\title{
Understanding parameter differences between analyses employing nested data subsets
}

\author{
Steven Gratton ${ }^{1,2 \star}$ and Anthony Challinor ${ }^{1,2,3} \dagger$ \\ ${ }^{1}$ Institute of Astronomy, Madingley Road, Cambridge CB3 OHA, UK \\ ${ }^{2}$ Kavli Institute for Cosmology Cambridge, Madingley Road, Cambridge CB3 OHA, UK \\ ${ }^{3}$ DAMTP, Centre for Mathematical Sciences, Wilberforce Road, Cambridge CB3 OWA, UK
}

18 November 2019

\begin{abstract}
We provide an analytical argument for understanding the likely nature of parameter shifts between those coming from an analysis of a dataset and from a subset of that dataset, assuming differences are down to noise and any intrinsic variance alone. This gives us a measure against which we can interpret changes seen in parameters and make judgements about the coherency of the data and the suitability of a model in describing those data.
\end{abstract}

Key words: methods: analytical - methods: statistical

\section{INTRODUCTION}

One would typically expect the posterior distributions of the parameters of a model to change as the datasets used to constrain them are changed. A part of this must be attributable to "scatter", i.e., noise and any intrinsic variance assumed by the model. However, it could also be an indication of a problem, either in the data (e.g., a systematic error in one of the datasets or an unaccounted-for relative calibration between two datasets) or in the model (i.e., the model is incomplete and unable to well-describe all of the data).

In this paper we provide an analytical argument for understanding the likely nature of parameter differences in the ideal, scatter-only, case. This gives us a diagnostic measure which we can use to interpret changes seen between parameters inferred from using subsets of the data. Thus we can make judgements about the internal coherency of the data and the appropriateness of a model for describing those data.

The method used here can also be used to derive simply other useful results for understanding differences between models and data. For example, Wilks (1938) derived the distribution that one should expect for the change in $\chi^{2}$ when allowing additional model parameters to vary if in fact they are unnecessary. In a cosmological context, this is very useful in knowing how strongly to take $\chi^{2}$ improvements as potential detections of systematics or new physics. In an appendix we show how the approach presented here can be used to rederive quickly this result.

Some of the techniques described in detail in this paper were used in Planck Collaboration et al. (2016a, 2017,

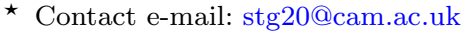

$\uparrow$ Contact e-mail: a.d.challinor@ast.cam.ac.uk
}

2020a). For similarly-motivated work see Raveri \& Hu (2019) and Lemos et al. (2020). The former paper discusses a number of "concordance/discordance estimators" within and between cosmological data sets, including some based on parameter shifts as here but assuming the data is Gaussiandistributed. The latter paper develops a "suspiciousness statistic" for "correlated" data sets but does not specifically investigate nested data subsets.

\section{DERIVATION OF MAIN RESULT}

Imagine one has a collection of data, denoted by the vector $\hat{\boldsymbol{X}}$. One has a parametric model in mind to describe these data, depending on a collection of parameters denoted by a vector $\boldsymbol{P}$. Let us write the probability for a realisation of the data to be within $d \boldsymbol{X}$ of $\boldsymbol{X}$ under the model as

$p(\boldsymbol{X}) d \boldsymbol{X}=e^{-S} d \boldsymbol{X}$,

where the "action" $S$ is a function of $\boldsymbol{X}$ and the parameters $\boldsymbol{P}$, and $d \boldsymbol{X}$ is the appropriate multi-dimensional measure on the data. (This form is motivated by analogy with the path integral approach to Euclidean quantum field theory, where $S$ plays the part of the Euclidean action.)

Let us now assume that the model can indeed describe the data and that the true parameter values are $\boldsymbol{P}_{0}$. Let us expand $S$ to second order in the parameters about $\boldsymbol{P}_{0}$ :

$$
\begin{aligned}
& S=S_{0}+\boldsymbol{S}^{T} \delta \boldsymbol{P}+\frac{1}{2} \delta \boldsymbol{P}^{T} \mathbf{S}^{\prime \prime} \boldsymbol{\delta} \boldsymbol{P}+\cdots \\
& \approx S_{0}+\boldsymbol{S}^{T} \boldsymbol{\delta} \boldsymbol{P}+\frac{1}{2} \delta \boldsymbol{P}^{T} \overline{\mathbf{S}^{\prime \prime}} \boldsymbol{\delta P} \\
& =\frac{1}{2}\left(\delta \boldsymbol{P}+{\overline{\mathbf{S}^{\prime \prime}}}^{-1} \boldsymbol{S}^{\prime}\right)^{T} \overline{\mathbf{S}^{\prime \prime}}\left(\delta \boldsymbol{P}+{\overline{\mathbf{S}^{\prime \prime}}}^{-1} \boldsymbol{S}^{\prime}\right)+\text { const. }
\end{aligned}
$$


Here $\boldsymbol{S}^{\prime}$ denotes the vector of derivatives of $S$ with respect to the parameters, superscript $T$ denotes transpose, and $\mathbf{S}^{\prime \prime}$ denotes the matrix of second derivatives. In the second line we have made the (typically-good) approximation of replacing $\mathbf{S}^{\prime \prime}$ with its average $\overline{\mathbf{S}^{\prime \prime}}$, where here and onwards an overline denotes an ensemble average of the indicated object with respect to data realizations from the assumed model with parameters $\boldsymbol{P}_{0}$. Equation (2) motivates the "maximumlikelihood" estimator for the parameters, and we now briefly recap some of its properties in preparation for what will follow. The fluctuation in parameters around $\boldsymbol{P}_{0}$ for any given realisation of the data is given by

$\delta \boldsymbol{P}=-{\overline{\mathbf{S}^{\prime \prime}}}^{-1} \boldsymbol{S}^{\prime}$,

where $\boldsymbol{S}^{\prime}$ is evaluated for the realization in question. This can be seen to be unbiased as follows. From Eq. (3) we have $\overline{\boldsymbol{\delta P}}=-{\overline{\mathbf{S}^{\prime \prime}}}^{-1} \overline{\boldsymbol{S}^{\prime}}$. Using Eq. (1) and considering, for example, the $i$ th parameter $P^{i}$, we have

$$
\begin{aligned}
\overline{S_{, i}} & =\int d X S_{, i} e^{-S} \\
& =-\int d X\left(e^{-S}\right)_{, i} \\
& =-\left(\int d X e^{-S}\right)_{, i} \\
& =-1, i=0,
\end{aligned}
$$

where $S_{, i}=\partial S / \partial P^{i}$. In the last line we have used the fact that the probability distribution for $X$ is normalized to unity. We see that $S_{, i}$ for the correct model averages to zero over realizations of that model, and so $\overline{\boldsymbol{\delta P}}=0$.

To obtain the covariance of the parameters we average the outer product of Eq. (3) over the ensemble. By judicious integrations by parts we can relate the average of this product of first derivatives to the average of a second derivative:

$$
\begin{aligned}
\overline{S_{, i} S_{, j}} & =\int d X S_{, i} S_{, j} e^{-S} \\
& =-\int d X S_{, i}\left(e^{-S}\right)_{, j} \\
& =-\left(\int d X S_{, i} e^{-S}\right)_{, j}+\int d X S_{, i j} e^{-S} \\
& =1_{, i j}+\overline{S_{, i j}} \\
& =\overline{S_{, i j}} .
\end{aligned}
$$

Hence

$$
\overline{\boldsymbol{\delta P} \delta \boldsymbol{P}^{T}}={\overline{\mathbf{S}^{\prime \prime}}}^{-1} \overline{\mathbf{S}^{\prime \prime}}{\overline{\mathbf{S}^{\prime \prime}}}^{-1}={\overline{\mathbf{S}^{\prime \prime}}}^{-1},
$$

the usual Fisher result.

Now let us imagine splitting our data $\hat{\boldsymbol{X}}$ into two pieces, $\hat{\boldsymbol{X}}_{1}$ and $\hat{\boldsymbol{X}}_{2}$, and performing an alternative parameter analysis using $\hat{\boldsymbol{X}}_{1}$ alone. The probability distribution we use for $\boldsymbol{X}_{1}$, described by $S_{1}$, must satisfy

$e^{-S_{1}} d \boldsymbol{X}_{1}=d \boldsymbol{X}_{1} \int d \boldsymbol{X}_{2} e^{-S}$

if the two analyses are to be consistent. Hence overlines for quantities involving $\boldsymbol{X}_{1}$ alone can equivalently be thought of as referring to averages over realisations of $\boldsymbol{X}_{1}$ alone or over the full data $\boldsymbol{X}$. Corresponding to Eq. (3) we have

$\delta \boldsymbol{P}_{1}=-{\overline{\mathbf{S}_{1}^{\prime \prime}}}^{-1} \boldsymbol{S}_{1}^{\prime}$, and corresponding to Eq. (6) we have

$\overline{\delta P_{1} \delta P_{1}^{T}}={\overline{\mathbf{S}_{1}^{\prime \prime}}}^{-1}$.

Now we are in a position to investigate the distribution of parameter differences, $\boldsymbol{\delta} \boldsymbol{P}_{1}-\boldsymbol{\delta} \boldsymbol{P}$, over the full ensemble. With each term averaging to zero, the parameter differences also average to zero. For the covariance, we have

$$
\begin{aligned}
& \overline{\left(\delta P_{1}-\delta P\right)\left(\delta P_{1}-\delta P\right)^{T}}=\overline{\mathbf{S}_{1}^{\prime \prime}}-1 \overline{S_{1}^{\prime} S_{1}^{\prime T}} \overline{\mathbf{S}_{1}^{\prime \prime}}-1 \\
& -{\overline{\mathbf{S}^{\prime \prime}}}^{-1} \overline{\mathbf{S}^{\prime} \mathbf{S}_{1}^{\prime T}} \overline{\mathbf{S}_{1}^{\prime \prime}}-1 \\
& -{\overline{\mathbf{S}_{1}^{\prime \prime}}}^{-1}{\overline{S_{1}^{\prime} S^{\prime T}}}_{\mathbf{S}^{\prime \prime}}^{-1} \\
& +{\overline{\mathbf{S}^{\prime \prime}}}^{-1} \overline{\boldsymbol{S}^{\prime} \boldsymbol{S}^{\prime T}}{\overline{\mathbf{S}^{\prime \prime}}}^{-1}
\end{aligned}
$$

and we see we need the average of the "mixed" quantity $\boldsymbol{S}_{1}^{\prime} \boldsymbol{S}^{\boldsymbol{T}}$. As with Eq. (5), using integrations by parts we can manipulate this into the form of the average of a second derivative:

$$
\begin{aligned}
\overline{S_{1, i} S_{, j}} & =\int d X S_{1, i} S_{, j} e^{-S} \\
& =-\int d X S_{1, i}\left(e^{-S}\right)_{, j} \\
& =-\int d X\left(S_{1, i} e^{-S}\right)_{, j}+\int d X S_{1, i j} e^{-S} \\
& =-\int d X_{1}\left(S_{1, i} \int d X_{2} e^{-S}\right)_{, j}+\int d X S_{1, i j} e^{-S} \\
& =-\left(\int d X_{1} S_{1, i} e^{-S_{1}}\right)_{, j}+\int d X S_{1, i j} e^{-S} \\
& =1_{, i j}+\overline{S_{1, i j}} \\
& =\overline{S_{1, i j}},
\end{aligned}
$$

where we have used the fact that by its definition $S_{1}$ must be independent of $X_{2}$. Hence we find

$\overline{\delta P_{1} \delta P^{T}}={\overline{\mathbf{S}^{\prime \prime}}}^{-1}$

the same as for $\overline{\boldsymbol{\delta} \boldsymbol{P} \boldsymbol{\delta} \boldsymbol{P}^{T}}$ itself. Substituting into Eq. (10) gives us the elegant final result

$\overline{\left(\boldsymbol{\delta} \boldsymbol{P}_{1}-\boldsymbol{\delta P}\right)\left(\boldsymbol{\delta} \boldsymbol{P}_{1}-\boldsymbol{\delta P}\right)^{T}}={\overline{\mathbf{S}_{1}^{\prime \prime}}}^{-1}-{\overline{\mathbf{S}^{\prime \prime}}}^{-1}$,

i.e., the covariance of the parameter differences between the partial and full analyses is the difference of their respective covariances.

\section{INTERPRETING DIFFERENCES IN MULTIPLE PARAMETERS}

For multiple parameters, one can form a " $\chi 2$ " for the differences in the parameters between the two analyses. This allows one to treat all parameters fairly, neither focussing on one outlier in particular nor neglecting degeneracies when judging how unlikely multiple shifts are. If we write the parameter shifts as $\boldsymbol{\Delta}=\boldsymbol{\delta} \boldsymbol{P}_{1}-\boldsymbol{\delta} \boldsymbol{P}$, in the Gaussian approxima- 
tion the probability $p(\boldsymbol{\Delta}) d \boldsymbol{\Delta}$ of being within $d \boldsymbol{\Delta}$ of $\boldsymbol{\Delta}$ is

$$
\begin{aligned}
& p(\Delta) d \boldsymbol{\Delta}= \\
& \frac{d \boldsymbol{\Delta}}{\left|2 \pi\left(\overline{\mathbf{S}}_{1}^{\prime \prime}-1-\overline{\mathbf{S}}^{-1}\right)\right|^{1 / 2}} \exp \left[-\frac{1}{2} \Delta^{T}\left({\overline{\mathbf{S}_{1}^{\prime \prime}}}^{-1}-{\overline{\mathbf{S}^{\prime \prime}}}^{-1}\right)^{-1} \Delta\right] .
\end{aligned}
$$

If the effective $\chi^{2}$ (i.e., minus twice the argument of the exponential) was to be large compared to the number of fitted model parameters then one might begin to worry about the fidelity of some aspect of the data taken as a whole or indeed about the applicability of the model to all of the data. The former would point to systematic effects, the latter to new physics.

\section{COSMIC MICROWAVE BACKGROUND EXAMPLE ANALYSIS}

Let us apply the formalism to an example drawn from cosmic microwave background (CMB) analysis in cosmology. Relevant introduction, motivation and definitions of the model parameters may be found in the Planck "cosmological parameters" series of papers (Planck Collaboration et al. 2014, 2016b, 2020b).

Here one takes maps of the microwave sky and compares them to predictions from a parameterized model, which we take here to be a standard six-parameter $\Lambda$ CDM cosmology. The model does not predict the actual pattern of fluctuations of the CMB, only their statistical properties. The primordial fluctuations in the early Universe are assumed to be Gaussian-distributed, giving an intrinsic "cosmic variance" to observations. The primordial 3D power spectrum is taken to change smoothly with scale and so is fully described by an amplitude and "tilt" parameter. In addition to temperature, "T", or intensity fluctuations, the linear polarization of the light also varies across the sky. In the simplest $\Lambda \mathrm{CDM}$ models that we shall consider here, this polarization can be described with the help of an additional scalar field, "E". From the T and E fields, there are three angular power spectra we can construct, the two auto-spectra $\hat{C}_{\ell}^{\mathrm{TT}}$ and $\hat{C}_{\ell}^{\mathrm{EE}}$ and the cross-spectrum $\hat{C}_{\ell}^{\mathrm{TE}}(\ell$ denoting the angular multipole). Neglecting Galactic and extragalactic contamination, and assuming the full sky is observed with no instrument noise, the negative of the log-likelihood of the observed spectra is

$-\ln L=(l+1 / 2)\left[\operatorname{tr}\left(\mathbf{C}_{\ell}^{-1} \hat{\mathbf{C}}_{\ell}\right)+\ln \left(\left|\mathbf{C}_{\ell}\right| /\left|\hat{\mathbf{C}}_{\ell}\right|\right)-1\right]$,

where

$\hat{\mathbf{c}}_{\ell}=\left(\begin{array}{ll}\hat{C}_{\ell}^{\mathrm{TT}} & \hat{C}_{\ell}^{\mathrm{TE}} \\ \hat{C}_{\ell}^{\mathrm{TE}} & \hat{C}_{\ell}^{\mathrm{EE}}\end{array}\right)$

and $\mathbf{C}_{\ell}$ is defined similarly but with the theory spectra.

Now it might be that an experiment is such that the polarization is observable only on larger angular scales (lower $\ell)$, whereas temperature measurements are possible down to finer angular scales (higher $\ell$ ). So we shall here investigate the shifts anticipated between a "full" measurement with TT, TE and EE spectra for angular multipoles $2 \leq \ell \leq 800$ and a "partial" one retaining the range $2 \leq \ell \leq 800$ for TT but only $2 \leq \ell \leq 29$ for TE and EE. (While somewhat arbitrary, these choices for the limits have been chosen to correspond to, e.g., the investigation of dependence of the parameters on multipole cuts in Efstathiou \& Gratton 2019. Very roughly speaking, while both Planck and the earlier WMAP satellite perform the "partial" spectral analysis, only Planck performs the "full" spectral analysis. So Planck's results and WMAP's results can be cross-checked against each other in the partial case, but we must turn to internal verifications within Planck of the new high- $\ell$ polarization data it adds.) This makes for a good test of our formalism since theory predicts the temperature and polarization signals to be correlated with each other.

We generate a fiducial power spectrum from a "best-fit" model of the Planck 2015 analysis (Planck Collaboration et al. 2016b) using CAMB (Lewis et al. 2000). Next, we generate a realization of the TT, TE and EE spectra from this model by first drawing Gaussian realizations of the $\mathrm{T}$ and $\mathrm{E}$ multipoles and forming their auto- and cross-power spectra. Finally, we perform Markov-chain Monte-Carlo (MCMC) analyses on both the full set of spectra and the partial set and find best-fit models in both cases using CosmoMC (Lewis \& Bridle 2002). Parameter means, standard deviations and best-fits are listed in Table 1 and 2D marginalised posterior distributions are illustrated in Fig. 1.

CosmoMC provides estimates of the covariance matrices for the posterior distributions derived from the MCMC chains. We use these on the right-hand side in Eq. (13) to obtain an estimate of the covariance of the parameter differences that we should expect to see. We are now able to compare predictions from this covariance to what is found in this test. To start with, from the diagonal of the matrix we derive standard deviations for the shifts in parameters between the full and partial analyses. We list in Table 2 these standard deviations, along with the measured shifts in both the best-fits and the means in terms of these standard deviations; the shifts are of the right magnitude to agree with the prediction.

Considering more than one parameter at a time, one might perform a singular-value-decomposition of Eq. (13) to identify the "most likely" shifts one should expect to see. One can also use the entire covariance of Eq. (13), as discussed in Sec. 3, to compute an overall "goodness-of-fit" for the shifts in all of the parameters. We obtain a $\chi^{2}$ of 9.84 for the shifts in the means (11.3 for shifts in best-fits) for our six degrees of freedom, a value greater than which would be expected about $13 \%(8 \%)$ of the time under the distribution in Eq. (14).

In computing the standard deviations of parameter shifts shown in Table 2 and associated $\chi^{2}$ values, we have used the covariances estimated from the MCMC chains, in a similar manner to what one would need to do in a real problem. In our simulation here, however, we know what the underlying model is and so can calculate $\overline{S_{, i j}}$ and $\overline{S_{1, i j}}$ analytically in terms of derivatives of the fiducial spectra with respect to the model parameters (these derivatives being evaluated numerically). Using these matrices, the standard deviations of the shifts change very little, but the $\chi^{2}$ of the shift in the means changes to 8.93 and the $\chi^{2}$ of the shift in the best-fits changes to 9.93 .

We go on to generate 100 further realizations of the power spectra, and find their best-fitting parameter values under the full and partial treatments. We compare the shifts 


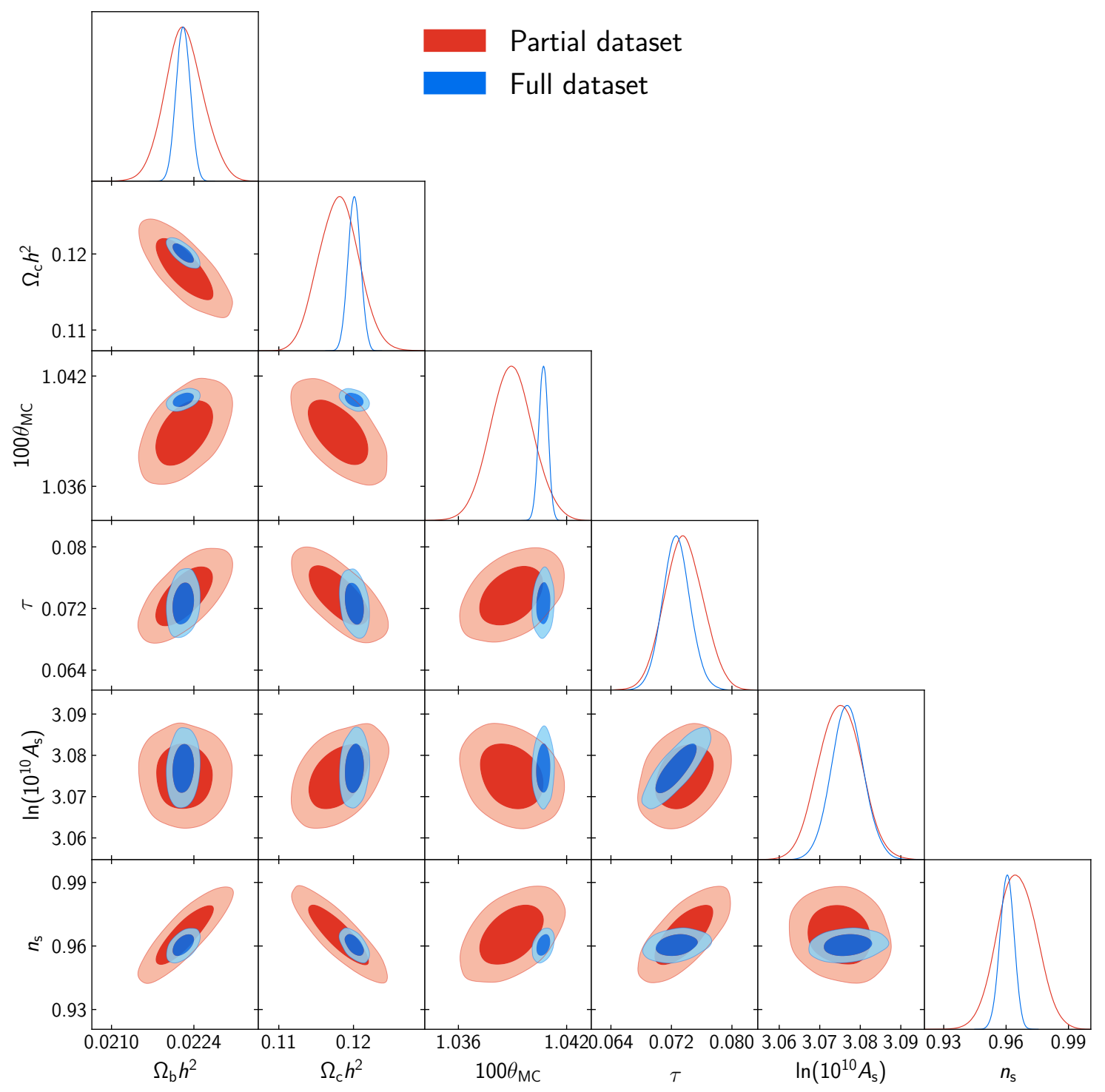

Figure 1. Two-dimensional marginalised posterior distributions for a full (blue; smaller contours) and a partial (red; larger contours) analysis of a simulated CMB dataset.

Table 1. Cosmological parameter constraints from a full and partial analysis of a simulated CMB data set (the posterior distributions are shown in Fig. 1). These results serve as our starting point for comparing parameter shifts to expectation.

\begin{tabular}{lcccccc}
\hline & \multicolumn{3}{c}{ Full analysis } & \multicolumn{3}{c}{ Partial analysis } \\
\hline & best fit & mean & std. dev. & best fit & mean & std. dev. \\
\hline$\Omega_{\mathrm{b}} h^{2}$ & 0.02221 & 0.02222 & 0.00012 & 0.02219 & 0.02224 & 0.00032 \\
$\Omega_{\mathrm{c}} h^{2}$ & 0.12013 & 0.12011 & 0.00083 & 0.11844 & 0.11804 & 0.00267 \\
$100 \Theta_{\mathrm{MC}}$ & 1.04070 & 1.04071 & 0.00025 & 1.03886 & 1.03895 & 0.00118 \\
$\tau$ & 0.07255 & 0.07265 & 0.00183 & 0.07337 & 0.07365 & 0.00254 \\
$\ln \left(10^{10} A_{\mathrm{s}}\right)$ & 3.07673 & 3.07689 & 0.00399 & 3.07529 & 3.07498 & 0.00522 \\
$n_{\mathrm{s}}$ & 0.96030 & 0.96040 & 0.00335 & 0.96384 & 0.96522 & 0.00937 \\
\hline
\end{tabular}

between the analyses to predictions from Eq. (13) in Fig. 2. We plot a histogram of the $\chi^{2}$ s of the shifts, using the analytic covariances, in Fig. 3 (performing a classic binned Pearson's chi-squared test comparing the histogram to the curve, we find a value of 9.5 for 10 effective degrees of freedom, indicating good agreement).
In order to obtain the good agreement with expectation shown, it was necessary to take into account the effect of "numerical noise" in the minimization procedure. The result returned by the CosmoMC minimizer routine is expected to be good to within a 0.05 -sigma tolerance of the true minimum for each parameter. Assuming such errors are independent 

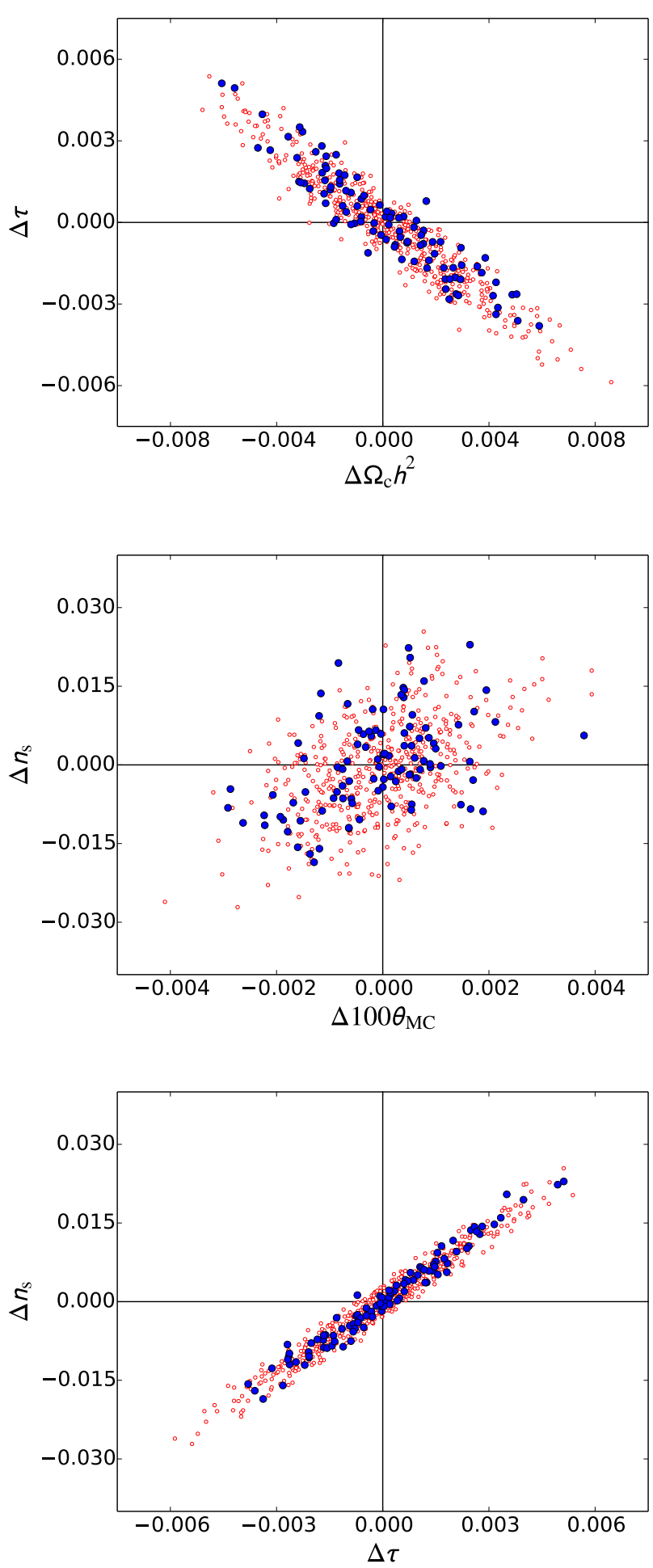

Figure 2. Three representative plots showing the agreement between parameter shifts found in simulations and those predicted from Eq. (13). The top plot shows shifts in $\tau$ against shifts in $\Omega_{\mathrm{c}} h^{2}$, the middle plot shows shifts in $n_{\mathrm{s}}$ against $100 \Theta_{\mathrm{MC}}$ and the bottom plot shows shifts in $n_{\mathrm{s}}$ against shifts in $\tau$. The large blue filled circles show the differences between parameters from the partial and full analyses for 100 simulated CMB datasets. The expectations from Eq. (13) are illustrated via 500 small red open circles, generated as multi-variate Gaussian realizations with that covariance.
Table 2. Comparison of the shifts in parameters between the two analyses reported in Table 1 to the predictions of Eq. (13). The first column shows the prediction of the expected standard deviations of the shifts in best-fitting parameters. The second and third columns show the shifts found, in units of these standard deviations, in the best-fits and posterior means respectively. For the simulation considered, the shifts are seen to be broadly in line with the predictions.

\begin{tabular}{lccc}
\hline & $\begin{array}{c}\text { std. dev. } \\
\text { of shift }\end{array}$ & $\begin{array}{c}\Delta \text { (best fit) in } \\
\text { shift std. dev.'s }\end{array}$ & $\begin{array}{c}\Delta \text { (mean) in } \\
\text { shift std. dev.'s }\end{array}$ \\
\hline$\Omega_{\mathrm{b}} h^{2}$ & 0.00030 & -0.10 & -0.06 \\
$\Omega_{\mathrm{c}} h^{2}$ & 0.00254 & -0.67 & -0.82 \\
$100 \Theta_{\mathrm{MC}}$ & 0.00115 & -1.59 & -1.53 \\
$\tau$ & 0.00175 & +0.47 & +0.57 \\
$\ln \left(10^{10} A_{\mathrm{s}}\right)$ & 0.00337 & -0.43 & -0.56 \\
$n_{\mathrm{s}}$ & 0.00875 & +0.40 & +0.55 \\
\hline
\end{tabular}

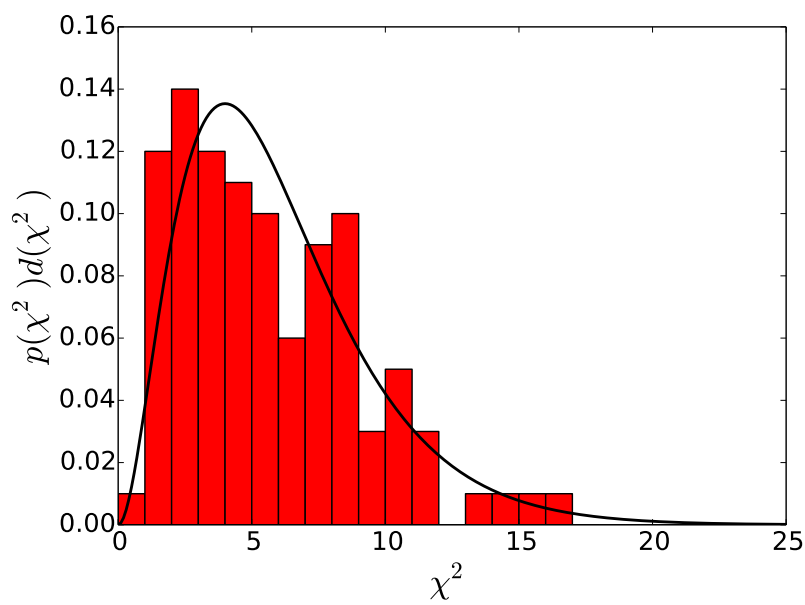

Figure 3. Normalized histogram showing the effective $\chi^{2}$ from Eq. (14) evaluated for the difference of best-fit parameters between the partial and full analyses of 100 simulated CMB datasets, using analytic covariances computed around the fiducial model (including terms accounting for tolerances in the minimization procedure), compared to a $\chi^{2}$ distribution for six degrees of freedom. (A classic binned Pearson's chi-squared test comparing the histogram to the curve confirms the visual impression that the two are in reasonable agreement with each other.)

between runs of the minimizer, their effects on the parameter differences can be accounted for by adding the covariances of the minimizer errors in each run to that of the difference. We therefore added $0.05^{2}$ times the diagonals of each of the full and partial covariances back to the covariance of the parameter differences. Without these terms a small number of the realizations appeared to have very unlikely shifts. In other analyses it would seem prudent to add analogous terms before considering $\chi^{2}$ for parameter shifts.

\section{COMMENTS AND EXTENSIONS}

Our result Eq. (13) should have a wide applicability, enabling one to compare analyses with differing combinations of datasets, e.g., to illuminate tensions between late-time measurements of the expansion rate of the Universe (see, 
e.g., Riess et al. 2018) and inferences from the $\Lambda$ CDM framework with Planck (see, e.g., the discussion in Planck Collaboration et al. 2020b), as well as comparing subsets of data taken with the same experiment.

We can understand generic features of parameter shifts from the form of Eq. (13), by considering how partial cancellations between the two covariance matrices may or may not occur as the constraining power between the two analyses changes. For example, if large parameter degeneracies that exist using the partial data set are broken using the full data set, then one would expect parameter differences to lie along those parameter degeneracies also. This is because the large degenerate eigenmode in the partial-analysis covariance matrix would not be significantly reduced by any corresponding mode in the full-analysis covariance matrix.

Note that in order to obtain the simple result of Eq. (13), we had to look at differences between one data combination and another "nested" within it. No such simple result exists in general for parameter differences between, say, two non-nested datasets $X_{1}$ and $X_{2}$. If the data sets happen to be independent, then our method does yield

$\overline{\left(\boldsymbol{\delta} \boldsymbol{P}_{1}-\delta \boldsymbol{P}_{2}\right)\left(\boldsymbol{\delta} \boldsymbol{P}_{1}-\delta \boldsymbol{P}_{2}\right)^{T}}={\overline{\mathbf{S}_{1}^{\prime \prime}}}^{-1}+{\overline{\mathbf{S}_{2}^{\prime \prime}}}^{-1}$,

with uncertainties now adding in quadrature as expected.

By applying Eq. (13) to multiple nested subsets, one can build up a "grand" covariance matrix for expectations of the parameter differences between all the analyses. Let parameters $\boldsymbol{P}_{2}$ come from an analysis involving a subset of the data that yielded parameters $\boldsymbol{P}_{1}$, itself from an analysis involving a subset of the data that yielded parameters $\boldsymbol{P}$. By considering appropriate conditional distributions associated with this covariance matrix, certain properties of the parameters obtained may be understood. For example, using Eqs. (6) and (12) we can compute the joint covariance of the differences $\boldsymbol{P}_{1}-\boldsymbol{P}$ and $\boldsymbol{P}_{2}-\boldsymbol{P}$ to compare their behaviour to expectation.

Our formalism can be used to investigate how parameter estimates should "track" towards the truth as more data is added. Imagine one happened to know what the true underlying parameters of a theory happened to be. Using Eqs. (6) and (12) and their analogues for subsets of subsets, one is able to construct the full covariance matrix for the fluctuations in parameters from the truth (i.e. the $\boldsymbol{\delta} \boldsymbol{P}, \boldsymbol{\delta} \boldsymbol{P}_{1}, \boldsymbol{\delta} \boldsymbol{P}_{2}, \ldots$ themselves). Treating these fluctuations to be Gaussian, one can then understand certain properties of the behaviour of the parameters by considering the associated lower-dimensional conditional distributions of the multi-dimensional Gaussian. For example, given $\boldsymbol{\delta} \boldsymbol{P}$, one's best estimate for $\boldsymbol{\delta} \boldsymbol{P}_{1}$ is just $\boldsymbol{\delta} \boldsymbol{P}$, whereas given $\boldsymbol{\delta} \boldsymbol{P}_{1}$, one should expect $\boldsymbol{\delta} \boldsymbol{P}$ to be $\mathbf{C C}_{1}^{-1} \boldsymbol{\delta} \boldsymbol{P}_{1}$ (with the $\mathbf{C}_{\text {s denoting re- }}$ spective covariance matrices). For a single parameter this reduces to $\sigma^{2} / \sigma_{1}^{2}$ times $\delta P_{1}$; as more data is added and uncertainties reduce, parameters are most likely to move monotonically closer to the truth. Given $\boldsymbol{P}$ and $\boldsymbol{P}_{2}$, one can show that $\boldsymbol{P}_{1}$ should peak around an intermediate value between the two. We can also gain some intuition about how the $\chi^{2}$ of fits should behave. Focusing on a single parameter for simplicity, given some $\delta P_{1}$ with a $\chi_{1}^{2}=\delta P_{1}^{2} / \sigma_{1}^{2}$, we should conditionally expect the fuller analysis to have a $\chi^{2}$ of $\sigma^{2} \chi_{1}^{2} / \sigma_{1}^{2}+\left(\sigma_{1}^{2}-\sigma^{2}\right) / \sigma_{1}^{2}$; if additional data is added that significantly reduces uncertainties, the significance of an outlier should decrease.
Finally, one can generalise the argument of Sec. 2 to include Gaussian priors on the parameters. However, the result is not as easily expressed in terms of the covariance matrices involved as it was in Eq. (13).

\section{CONCLUSIONS}

In this note we have presented arguments aimed to aid the understanding of relations between inferences using full and partial amounts of data and, in the appendix, between inferences using standard and extended models.

Our work provides some insight into the sorts of posterior variations one might expect when comparing related Bayesian parameter estimations. A "rule of thumb" for a parameter that is well-constrained by the data (so that any effect of priors may be neglected) is as follows: when more data is added, a significant change in the width of the posterior distribution will be accompanied by a difference in the most likely parameter value that can be a significant fraction of the larger width. If the posteriors have similar widths then there should be little shift in the peak position.

\section{ACKNOWLEDGEMENTS}

We thank George Efstathiou, Antony Lewis and the Planck Parameters and Likelihood teams for helpful comments and discussions over the development of this work. We thank the anonymous referee of this paper for useful comments on the original manuscript.

Our MCMC analyses were performed using the Cambridge Service for Data Driven Discovery (CSD3) operated by the University of Cambridge Research Computing Service (http://www.csd3.cam.ac.uk/), provided by Dell EMC and Intel using Tier-2 funding from the Engineering and Physical Sciences Research Council, and DiRAC funding from the Science and Technology Facilities Council (https://dirac.ac.uk).

SG and AC acknowledge support from the UK Science and Technology Facilities Council (grant numbers ST/N000927/1 and ST/S000623/1).

\section{APPENDIX A: WILKS' THEOREM}

Imagine we fit a model with $n_{a}$ parameters to our data, and then fit an extended model with an additional $n_{b}$ parameters. In the case where the first model is the correct one, i.e., the additional parameters are not required, by how much should we expect the extended model to improve the goodness of fit by chance? For a cosmological example, one might allow the amplitude of the $3 \mathrm{D}$ power spectrum of primordial gravitational waves to vary in the extended model when they are actually negligible. Wilks' Theorem (Wilks 1938) addresses expectations for the improvement in the loglikelihood for the wider analysis over the more specific one. The theorem shows the improvement to be $\chi^{2}$-distributed with a number of degrees of freedom equal to the number $n_{b}$ of additional parameters the wider analysis has over the more specific one.

Equations (2) and (3) can be used to rederive this result. Here we need to keep track of the constant to evaluate the 
action $S$ at the best-fit model (denoted with subscript "bf" for "best-fit"):

$$
\begin{aligned}
S_{\mathrm{bf}} & \approx S_{0}-\frac{1}{2} \boldsymbol{S}^{\prime T}{\overline{\mathbf{S}^{\prime \prime}}}^{-1} \boldsymbol{S}^{\prime} \\
& =S_{0}-\frac{1}{2} \boldsymbol{\delta} \boldsymbol{P}^{T} \overline{\mathbf{S}^{\prime \prime}} \boldsymbol{\delta} \boldsymbol{P},
\end{aligned}
$$

where we have used Eq. (3) and understand the parameter shifts $\boldsymbol{\delta} \boldsymbol{P}$ in the second line to be between the best-fit model of the class considered and the underlying one.

We split our $\boldsymbol{\delta} \boldsymbol{P}$ into two parts, $\boldsymbol{\delta} \boldsymbol{P}_{a}$ and $\boldsymbol{\delta} \boldsymbol{P}_{b}$, corresponding to the usual parameters and the additional parameters, respectively, of lengths $n_{a}$ and $n_{b}$. For the restricted analysis, indicated by superscript "R", we have

$\delta \boldsymbol{P}_{a}^{\mathrm{R}}=-{\overline{\mathbf{S}_{a a}^{\prime \prime}}}^{-1} \boldsymbol{S}_{a}^{\prime}$,

where we have partitioned $\boldsymbol{S}^{\prime}$ and $\mathbf{S}^{\prime \prime}$ as

$$
\begin{aligned}
\boldsymbol{S}^{\prime} & =\left(\begin{array}{l}
\boldsymbol{S}_{a}^{\prime} \\
\boldsymbol{S}_{b}^{\prime}
\end{array}\right), \\
\mathbf{S}^{\prime \prime} & =\left(\begin{array}{ll}
\mathbf{S}_{a a}^{\prime \prime} & \mathbf{S}_{a b}^{\prime \prime} \\
\mathbf{S}_{b a}^{\prime \prime} & \mathbf{S}_{b b}^{\prime \prime}
\end{array}\right) .
\end{aligned}
$$

Let us introduce an $n$-by- $n_{a}$ projection matrix $\mathbf{M}$ (with $n=$ $n_{a}+n_{b}$, corresponding to the total number of parameters varied in the wider analysis),

$\mathbf{M}=\left(\begin{array}{l}\mathbf{I}_{a a} \\ \mathbf{0}_{b a}\end{array}\right)$

where $\mathbf{I}_{a a}$ denotes the $n_{a}$-by- $n_{a}$ identity matrix and $\mathbf{0}_{b a}$ denotes an $n_{b}$-by- $n_{a}$ matrix of zeros. This allows us to express $S_{a \mathrm{bf}}-S_{0}$ for the usual model, $S_{a \mathrm{bf}}$ being its best-fit action, as

$$
\begin{aligned}
S_{a \mathrm{bf}}-S_{0} & =-\frac{1}{2}\left(\boldsymbol{\delta} \boldsymbol{P}_{a}^{\mathrm{R}}\right)^{T} \overline{\mathbf{S}_{a a}^{\prime \prime}} \boldsymbol{\delta} \boldsymbol{P}_{a}^{\mathrm{R}} \\
& =-\frac{1}{2} \boldsymbol{S}_{a}^{\prime T}{\overline{\mathbf{S}_{a a}^{\prime \prime}}}^{-1} \boldsymbol{S}_{a}^{\prime} \\
& =-\frac{1}{2} \boldsymbol{S}^{\prime T} \mathbf{M}{\overline{\mathbf{S}_{a a}^{\prime \prime}}}^{-1} \mathbf{M}^{T} \boldsymbol{S}^{\prime},
\end{aligned}
$$

i.e., in terms of $\boldsymbol{S}^{\prime}$ rather than just $\boldsymbol{S}_{a}^{\prime}$. Subtracting this from the same quantity evaluated for the extended model yields

$$
\begin{aligned}
S_{\mathrm{bf}}-S_{a \mathrm{bf}} & =-\frac{1}{2} \boldsymbol{S}^{\prime T}\left({\overline{\mathbf{S}^{\prime \prime}}}^{-1}-\mathbf{M}{\overline{\mathbf{S}_{a a}^{\prime \prime}}}^{-1} \mathbf{M}^{T}\right) \boldsymbol{S}^{\prime} \\
& =-\frac{1}{2} \boldsymbol{\delta} \boldsymbol{P}^{T}\left(\overline{\mathbf{S}^{\prime \prime}}-\overline{\mathbf{S}^{\prime \prime}} \mathbf{M} \overline{\mathbf{S}_{a a}^{\prime \prime}}-1 \mathbf{M}^{T} \overline{\mathbf{S}^{\prime \prime}}\right) \boldsymbol{\delta P},
\end{aligned}
$$

an expression now involving only $\boldsymbol{\delta} \boldsymbol{P}$, the shift from the underlying model to the extended best-fit model, and not also $\boldsymbol{\delta} \boldsymbol{P}_{a}$. Using Eq. (A4) the bracketed term becomes

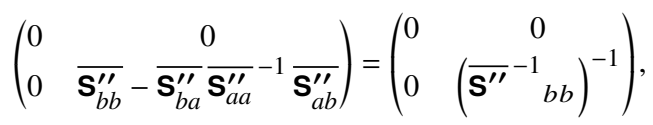

with ${\overline{\mathbf{S}^{\prime \prime}}}^{-1} b b$ being the lower-right block of the inverse of $\overline{\mathbf{S}^{\prime \prime}}$. Hence

$$
S_{\mathrm{bf}}-S_{a \mathrm{bf}}=-\frac{1}{2} \boldsymbol{\delta} \boldsymbol{P}_{b}^{T}\left({\overline{\mathbf{s}^{\prime \prime}}}^{-1} b b\right)^{-1} \boldsymbol{\delta} \boldsymbol{P}_{b}
$$

which we note only depends on the additional parameters $\boldsymbol{\delta} \boldsymbol{P}_{b}$. So to understand how the action is distributed, we need to know how the $\boldsymbol{\delta} \boldsymbol{P}_{b}$ are distributed.

From Eq. (6), we see that the $\boldsymbol{\delta} \boldsymbol{P}$ have covariance ${\overline{\mathbf{S}^{\prime \prime}}}^{-1}$, and hence the $\boldsymbol{\delta} \boldsymbol{P}_{b}$ have covariance ${\overline{\mathbf{S}^{\prime \prime}}}^{-1} b b$. But this is just the same matrix as appears in the right-hand side of Eq. (A9). So, to the extent that the parameter shifts may be approximated as Gaussian-distributed about the fiducial model, we can immediately recognize $-2\left(S_{\mathrm{bf}}-S_{a \mathrm{bf}}\right)$ to be $\chi^{2}$-distributed with $n_{b}$ degrees of freedom as in Wilks (1938).

\section{DATA AVAILABILITY}

The simulated data, MCMC chains and minimizer runs used in Sec. 4 can be made available to interested parties by email request to the authors.

\section{REFERENCES}

Efstathiou G., Gratton S., 2019, arXiv e-prints, p. arXiv:1910.00483

Lemos P., Köhlinger F., Handley W., Joachimi B., Whiteway L., Lahav O., 2020, MNRAS, 496, 4647

Lewis A., Bridle S., 2002, Phys. Rev. D, 66, 103511

Lewis A., Challinor A., Lasenby A., 2000, ApJ, 538, 473

Planck Collaboration et al., 2014, A\&A, 571, A16

Planck Collaboration et al., 2016a, A\&A, 594, A11

Planck Collaboration et al., 2016b, A\&A, 594, A13

Planck Collaboration et al., 2017, A\&A, 607, A95

Planck Collaboration et al., 2020a, A\&A, 641, A5

Planck Collaboration et al., 2020b, A\&A, 641, A6

Raveri M., Hu W., 2019, Phys. Rev. D, 99, 043506

Riess A. G., et al., 2018, ApJ, 855, 136

Wilks S. S., 1938, Ann. Math. Statist., 9, 60

This paper has been typeset from a $\mathrm{T}_{\mathrm{E}} \mathrm{X} / \mathrm{LAT} \mathrm{T}$ file prepared by the author. 